\title{
Correction to: Primary Sjögren's syndrome (pSS)-related cerebellar ataxia: a systematic review and meta-analysis
}

\author{
Andreas Liampas $^{1,2} \cdot$ Antonios Nteveros $^{1} \cdot$ Konstantinos Parperis $^{1} \cdot$ Mohammed Akil $^{3} \cdot$ Efthymios Dardiotis $^{4}$. \\ Elizabeth Andreadou ${ }^{5} \cdot$ Marios Hadjivassiliou $^{6} \cdot$ Panagiotis Zis $^{1}$ (i)
}

(c) Belgian Neurological Society 2021

\section{Correction to: Acta Neurologica Belgica https://doi.org/10.1007/s13760-021-01784-1}

The first name and family name of all authors have been incorrectly swapped in the original publication. The complete correct author names are given below.

Andreas Liampas, Antonios Nteveros, Konstantinos Parperis, Mohammed Akil, Efthymios Dardiotis, Elizabeth Andreadou, Marios Hadjivassiliou, Panagiotis Zis.

The original article has been corrected.
The original article can be found online at https://doi.org/10.1007/ s13760-021-01784-1.

\section{Panagiotis Zis}

takiszis@gmail.com

Andreas Liampas

liampasand@gmail.com

Antonios Nteveros

nteveros.antonios@ucy.ac.cy

Konstantinos Parperis

parperis.konstantinos-marinos@ucy.ac.cy

Mohammed Akil

m.akil@sheffield.ac.uk

Efthymios Dardiotis

edar@med.uth.gr

Elizabeth Andreadou

eandread@med.uoa.gr

Marios Hadjivassiliou

m.hadjivassiliou@ sheffield.ac.uk
Publisher's Note Springer Nature remains neutral with regard to jurisdictional claims in published maps and institutional affiliations.
1 Medical School, University of Cyprus, Nicosia, Cyprus

2 Department of Neurology, Nicosia General Hospital, Nicosia, Cyprus

3 Rheumatology Department, Sheffield Teaching Hospitals NHS Trust, Sheffield, UK

4 Department of Neurology, Faculty of Medicine, School of Health Sciences, University of Thessaly, University Hospital of Larissa, Larissa, Greece

5 First Department of Neurology, National and Kapodistiran University of Athens, School of Medicine, "Eginition" University Hospital, Athens, Greece

6 Academic Department of Neurosciences, Sheffield Teaching Hospitals NHS Foundation Trust, Sheffield, UK 